\title{
Dynamical Equation and Monte Carlo Simulation of the Two-time Wigner Function for Electron Quantum Transport
}

\author{
R. BRUNETTI*, A. BERTONI, P. BORDONE and C. JACOBONI \\ Istituto Nazionale per la Fisica della Materia, Dipartimento di Fisica, Universita' di Modena e Reggio Emilia, \\ via Campi 213/A, I-41100 Modena, Italy
}

\begin{abstract}
Within the Wigner-function formalism for electron quantum transport in semiconductors a two-time Wigner function is defined starting from the Green-function formalism. After a proper Fourier transform a Wigner function depending on $p$ and $\omega$ as independent variables is obtained. This new Wigner function extends the Wigner formalism to the frequency domain and carries information related to the spectral density of the system. A Monte Carlo approach based on the generation of Wigner paths, already developed for the single-time Wigner function, has been extended to evaluate the momentum and energy-dependent Wigner function. Results will be shown for electrons subject to the action of an external field and in presence of scattering with optical phonons.
\end{abstract}

Keywords: Two-time Wigner function; Wigner paths; Quantum transport; Quantum Monte Carlo procedure

\section{INTRODUCTION}

The Wigner formulation of quantum mechanics based on the concept of Wigner function (WF) proved to be particularly suitable for the study of quantum transport in mesoscopic systems due to the many analogies which can be established with the semiclassical transport theory [1]. Owing to the possibility of comparing in many cases quantum with classical results quantum effects can be discussed in a physically intuitive but rigorous theoretical framework [2].

Furthermore this formalism allows to describe quantum transport in open systems [3] and the quantum dynamical equation for the WF can be solved by means of a Monte Carlo procedure based on the generation of Wigner paths (WP) [4]. At present, due to the heavy computational burden required by the numerical algorithm, only paths with few scattering processes can be accounted for.

*Corresponding author. Tel.: +39059 2055277, Fax: +39059 367488, e-mail: brunetti.rossella@unimo.it 
This computational limitation however is not too severe for quantum transport in mesoscopic systems, where carriers crossing the regions of interest experience few scattering events anyway.

A generalized WF that includes the phonon states has been introduced [5] and the electron WF is obtained from it by performing a trace over the phonon variables. When the dynamical equation for the electron WF is solved, due to the electronphonon interaction non-diagonal states in the phonon variables must be included since the trace does not commute with the interaction hamiltonian [5].

$$
G^{<}\left(\boldsymbol{r}_{1}, t_{1} ; \boldsymbol{r}_{2}, t_{2}\right)=\frac{i}{\hbar}\left\langle\Psi^{\dagger}\left(\boldsymbol{r}_{2}, t_{2}\right) \Psi\left(\boldsymbol{r}_{1}, t_{1}\right)\right\rangle
$$

where $\Psi$ and $\Psi^{\dagger}$ the electron annihilation and creation field operators, and \langle\rangle indicates quantum and ensemble average. If electrons and phonons are considered, the wave function describing the system of interest is $\Phi\left(\boldsymbol{r},\left\{n_{q}\right\}, t\right)=$ $\left\langle\boldsymbol{r},\left\{n_{q}\right\} \mid \Phi(t)\right\rangle$, where $\left\{n_{q}\right\}$ is the set of quantum numbers in the occupation-number representation for the phonon gas. After some straightforward manipulations of the above equations we get for $\mathbb{G}^{<}:$

$$
G^{<}\left(\boldsymbol{r}_{1}, t_{1} ; \boldsymbol{r}_{2}, t_{2}\right)=\frac{i}{\hbar} \sum_{\left\{n_{q}\right\}} \sum_{\left\{n_{q}^{\prime}\right\}}\left\langle 0\left|\overline{\prod_{q} \frac{1}{\sqrt{n_{q} !}}\left(a_{q}\right)^{n_{q}} \Phi^{*}\left(\boldsymbol{r}_{2},\left\{n_{q}\right\}, t_{2}\right) U\left(t_{2}, t_{1}\right) \Phi\left(\boldsymbol{r}_{1},\left\{n_{q}^{\prime}\right\}, t_{1}\right) \prod_{q} \frac{1}{\sqrt{n_{q}^{\prime} !}}\left(a_{q}^{\dagger}\right)^{n_{q}^{\prime}}}\right| 0\right\rangle
$$

The theoretical developments summarized above have been analysed for the single-time WF obtained from the corresponding single-time density operator, which is appropriate to analyse transport properties like current density or electronic diffusion.

If information about the energy spreading of the system is of interest, then a generalization of the Wigner approach must be developed starting from the two-time Green function $G^{<}$.

This paper presents the above theoretical development (Sect. 2) and it shows that the concept of Wigner path can still be applied to calculate the energy and momentum-dependent WF from a Monte Carlo simulation (Sect. 3). Some results are also shown at the end of the paper (Sect. 4).

\section{THEORETICAL APPROACH}

\subsection{Definition of the Frequency-dependent Wigner Function}

The theoretical approach starts from the electron Green function $\mathrm{G}^{<}$:
The evolution operator $U$ is obtained from the hamiltonian $\mathbf{H}$ of the system which can be written, in our case, as: $\mathbb{H}=\mathbb{H}_{e}+\mathbb{H}_{p}+\mathbb{H}_{e p}$ where the three terms describe the electron and phonon hamiltonians and the interaction term, respectively. If the evolution operator in Eq. (2) is due to the above Hamiltonian, then:

$$
\begin{aligned}
& G^{<}\left(\boldsymbol{r}_{1}, t_{1} ; \boldsymbol{r}_{2}, t_{2}\right)
\end{aligned}
$$

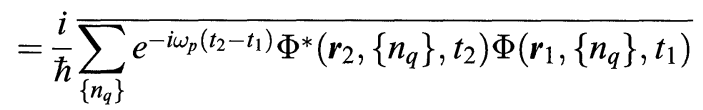

$$
\begin{aligned}
& =\frac{i}{\hbar} \overline{\sum_{\left\{n_{q}\right\}} g^{*}\left(\boldsymbol{r}_{2},\left\{n_{q}\right\}, t_{2}\right) g\left(\boldsymbol{r}_{1},\left\{n_{q}\right\}, t_{1}\right)}
\end{aligned}
$$

where $g\left(\boldsymbol{r},\left\{n_{q}\right\}, t\right)=e^{i \omega\left(\left\{n_{q}\right\}\right) t} \Phi\left(\boldsymbol{r},\left\{n_{q}\right\}, t\right)$ is the wave function of the system, where the time dependence due to free phonons has been eliminated by the exponential factor.

Using the conventional new set of space-time variables: $t=\left(t_{1}+t_{2}\right) / 2, \quad \tau=t_{2}-t_{1}, \quad \boldsymbol{r}=\left(\boldsymbol{r}_{1}+\boldsymbol{r}_{2}\right) / 2$, $\boldsymbol{r}^{\prime}=\boldsymbol{r}_{2}-\boldsymbol{r}_{1}$ and applying a Fourier transform we 
obtain the following WF:

$$
\begin{aligned}
& f_{w}(\boldsymbol{r}, \boldsymbol{p}, t, \omega)=-i \hbar G^{<}(\boldsymbol{r}, \boldsymbol{p}, t, \omega) \\
&= \sum_{\left\{n_{q}\right\}} \int_{-\infty}^{\infty} e^{i p \boldsymbol{r}^{\prime} / \hbar} \\
& \quad \times d \boldsymbol{r}^{\prime} \int_{-\infty}^{\infty} e^{-i \omega \tau} d \tau \frac{}{g\left(\boldsymbol{r}-\boldsymbol{r}^{\prime} / 2,\left\{n_{q}\right\}, t-\tau / 2\right)} \\
& g^{*}\left(\boldsymbol{r}+\boldsymbol{r}^{\prime} / 2,\left\{n_{q}\right\}, t+\tau / 2\right)
\end{aligned}
$$

We can finally extend the above equation to describe a system which is not diagonal on the phonon variables as follows:

$$
\begin{aligned}
f_{w}\left(\boldsymbol{r}, \boldsymbol{p},\left\{n_{q}\right\},\left\{n_{q}^{\prime}\right\}, t, \omega\right)= \\
=\int_{-\infty}^{\infty} e^{i p \boldsymbol{r}^{\prime} / \hbar} d \boldsymbol{r}^{\prime} \\
\quad \times \int_{-\infty}^{\infty} e^{-i \omega \tau} d \tau \frac{}{g^{*}\left(\boldsymbol{r}+\boldsymbol{r}^{\prime} / 2,\left\{n_{q}^{\prime}\right\}, t+\tau / 2\right)}
\end{aligned}
$$

As for the single-time WF, we have:

$$
f_{w}(\boldsymbol{r}, \boldsymbol{p}, t, \omega)=\sum_{\left\{n_{q}\right\}} f_{w}\left(\boldsymbol{r}, \boldsymbol{p},\left\{n_{q}\right\},\left\{n_{q}\right\}, t, \omega\right) .
$$

\subsection{Dynamics of the Frequency-dependent Wigner Function}

The dynamical evolution equation for the WF in the above equation is:

$$
\begin{aligned}
\frac{\partial}{\partial t} f_{w}\left(\boldsymbol{r}, \boldsymbol{p},\left\{n_{q}\right\},\left\{n_{q}^{\prime}\right\}, t, \omega\right)= \\
=\int_{-\infty}^{\infty} e^{i \boldsymbol{p} \boldsymbol{r}^{\prime} / \hbar} d \boldsymbol{r}^{\prime} \int_{-\infty}^{\infty} e^{-i \omega \tau} d \tau \\
\quad \times \overline{\left\{\left[\frac{\partial}{\partial t} g\left(\boldsymbol{r}-\boldsymbol{r}^{\prime} / 2,\left\{n_{q}\right\}, t-\tau / 2\right)\right]\right.} \\
\left.g^{*}\left(\boldsymbol{r}+\boldsymbol{r}^{\prime} / 2,\left\{n_{q}^{\prime}\right\}, t+\tau / 2\right)+g \frac{\partial}{\partial t} g^{*}\right\}
\end{aligned}
$$

By calculating the time derivative of $g$ and $g^{*}$ the terms coming from the free-phonon dynamics can be eliminated yielding:

$$
\begin{aligned}
\frac{\partial}{\partial t} f_{w}\left(\boldsymbol{r}, \boldsymbol{p},\left\{n_{q}\right\},\left\{n_{q}^{\prime}\right\}, t, \omega\right)= \\
=\frac{\int_{-\infty}^{\infty} e^{i p \boldsymbol{r}^{\prime} / \hbar} d \boldsymbol{r}^{\prime} \int_{-\infty}^{\infty} e^{-i \omega \tau} d \tau \frac{1}{i \hbar}}{\left\{\begin{array}{c}
{\left[\left(\mathbb{H}_{e}+\mathbb{H}_{e p}\right) g\left(\boldsymbol{r}-\boldsymbol{r}^{\prime} / 2,\left\{n_{q}\right\}, t-\tau / 2\right)\right]} \\
g^{*}\left(\boldsymbol{r}+\boldsymbol{r}^{\prime} / 2,\left\{n_{q}^{\prime}\right\}, t+\tau / 2\right) \\
-g\left(\boldsymbol{r}-\boldsymbol{r}^{\prime} / 2,\left\{n_{q}\right\}, t-\tau / 2\right)\left[\left(\mathbb{H}_{e}+\mathbb{H}_{e p}\right)\right. \\
\left.g\left(\boldsymbol{r}+\boldsymbol{r}^{\prime} / 2,\left\{n_{q}^{\prime}\right\}, t+\tau / 2\right)\right]^{*}
\end{array}\right\}}
\end{aligned}
$$

The electron hamiltonian $\mathbb{H}_{\mathbb{E}}=\mathbb{U}_{\mathbb{\Phi}}+\mathbb{H}_{\mathbb{E}}+\mathbb{V}$ is the sum of the kinetic term $\mathbb{H}_{\mathbb{E}}$, the external field term $\mathbb{H}_{\mathbb{E}}$, and a term associated to a non-smooth potential profile like, for example, the band offset of a heterostructure. The effect of the three terms on the WF dynamics can be studied separately.

The mathematical manipulation of the kinetic term provides the same result obtained for the traditional WF [1]:

$$
\left.\frac{\partial}{\partial t}\right|_{o} f_{w}\left(\boldsymbol{r}, \boldsymbol{p},\left\{n_{q}\right\},\left\{n_{q}^{\prime}\right\}, t, \omega\right)=-\frac{\boldsymbol{p}}{m} \nabla_{r} f_{w}
$$

The contribution of any potential V(r) to the WF dynamics has the general form:

$$
\begin{aligned}
& \left.\frac{\partial}{\partial t}\right|_{V} f_{w}\left(\boldsymbol{r}, \boldsymbol{p},\left\{n_{q}\right\},\left\{n_{q}^{\prime}\right\}, t, \omega\right) \\
& \quad=\int_{-\infty}^{\infty} d \boldsymbol{p}^{\prime} V_{w}\left(\boldsymbol{r}, \boldsymbol{p}-\boldsymbol{p}^{\prime}\right) f_{w}\left(\boldsymbol{r}, \boldsymbol{p}^{\prime},\left\{n_{q}\right\},\left\{n_{q}^{\prime}\right\}, t, \omega\right)
\end{aligned}
$$

where

$$
\begin{aligned}
V_{w}(\boldsymbol{r}, \boldsymbol{p})= & \frac{1}{i \hbar} \frac{1}{(2 \pi \hbar)^{3}} \\
& \int d \boldsymbol{r}^{\prime} e^{i \boldsymbol{p} \boldsymbol{r}^{\prime} / \hbar}\left[V\left(\boldsymbol{r}-\boldsymbol{r}^{\prime} / 2\right)-V\left(\boldsymbol{r}+\boldsymbol{r}^{\prime} / 2\right)\right]
\end{aligned}
$$

The same result applies for the potential term $\mathbb{H}_{\mathbb{E}}$ due to external fields. In the simple case of a constant or harmonic force, however, the above 
term reduces to:

$$
\begin{aligned}
& \left.\frac{\partial}{\partial t}\right|_{F} f_{w}\left(\boldsymbol{r}, \boldsymbol{p},\left\{n_{q}\right\},\left\{n_{q}^{\prime}\right\}, t, \omega\right) \\
& \quad=\nabla V(\boldsymbol{r}) \nabla_{p} f_{w}\left(\boldsymbol{r}, \boldsymbol{p},\left\{n_{q}\right\},\left\{n_{q}^{\prime}\right\}, t, \omega\right)=-\boldsymbol{F} \frac{\partial}{\partial \boldsymbol{p}} f_{w}
\end{aligned}
$$

The manipulation of the contribution to the time evolution of the WF due to electron-phonon hamiltonian

$$
\mathbb{H}_{e q}=i \hbar \sum_{q^{\prime}} F\left(\boldsymbol{q}^{\prime}\right)\left(b_{q^{\prime}} e^{i \boldsymbol{q}^{\prime} \boldsymbol{r}}-b_{q^{\prime}}^{\dagger} e^{-i \boldsymbol{q}^{\prime} \boldsymbol{r}}\right)
$$

(where $\mathrm{F}$ is a real function) requires more attention.

The contribution to the time evolution of the WF coming from this term has the form:

$$
\begin{aligned}
& \left.\frac{\partial}{\partial t}\right|_{e p} f_{w}\left(\boldsymbol{r}, \boldsymbol{p},\left\{n_{q}\right\},\left\{n_{q}^{\prime}\right\}, t, \omega\right) \\
& =\int_{-\infty}^{\infty} e^{i p r^{\prime} / \hbar} d \boldsymbol{r}^{\prime} \int_{-\infty}^{\infty} e^{-i \omega \tau} d \tau \sum_{\boldsymbol{q}^{\prime}} F\left(\boldsymbol{q}^{\prime}\right) \\
& \left\{\overline{\left.\mathbb{W}_{\boldsymbol{q}^{\prime}} e^{i \boldsymbol{q}^{\prime}\left(\boldsymbol{r}-\boldsymbol{r}^{\prime} / 2\right)} g\left(\boldsymbol{r}-\boldsymbol{r}^{\prime} / 2\right),\left\{n_{q}\right\}, t-\tau / 2\right)}\right. \\
& g^{*}\left(\boldsymbol{r}+\boldsymbol{r}^{\prime} / 2,\left\{n_{q}^{\prime}\right\}, t+\tau / 2\right) \\
& -\overline{\left.\mathbb{b}_{\boldsymbol{q}^{\prime}}^{\dagger} e^{-i \boldsymbol{q}^{\prime}\left(\boldsymbol{r}-\boldsymbol{r}^{\prime} / 2\right)} g\left(\boldsymbol{r}-\boldsymbol{r}^{\prime} / 2\right),\left\{n_{q}\right\}, t-\tau / 2\right)} \\
& g^{*}\left(\boldsymbol{r}+\boldsymbol{r}^{\prime} / 2,\left\{n_{q}^{\prime}\right\}, t+\tau / 2\right) \\
& +\overline{\left.g\left(\boldsymbol{r}-\boldsymbol{r}^{\prime} / 2\right),\left\{n_{q}\right\}, t-\tau / 2\right)\left[\mathbb{W}_{\boldsymbol{q}^{\prime}} e^{i \boldsymbol{q}^{\prime}\left(\boldsymbol{r}+\boldsymbol{r}^{\prime} / 2\right)}\right.} \\
& \left.g\left(\boldsymbol{r}+\boldsymbol{r}^{\prime} / 2,\left\{n_{q}^{\prime}\right\}, t+\tau / 2\right)\right]^{*} \\
& -\overline{\left.g\left(\boldsymbol{r}-\boldsymbol{r}^{\prime} / 2\right),\left\{n_{q}\right\}, t-\tau / 2\right)\left[\mathbb{b}_{q^{\prime}}^{\dagger} e^{-i \boldsymbol{q}^{\prime}\left(\boldsymbol{r}+\boldsymbol{r}^{\prime} / 2\right)}\right.} \\
& \left.\left.g\left(\boldsymbol{r}+\boldsymbol{r}^{\prime} / 2,\left\{n_{q}^{\prime}\right\}, t+\tau / 2\right)\right]^{*}\right\}
\end{aligned}
$$

By applying the phonon creation and annihilation operators $b_{q}$ and $b_{q}^{\dagger}$ to the function $g$, after some calculations which must be omitted here for brevity, we get:

$$
\begin{aligned}
& \left.\frac{\partial}{\partial t}\right|_{e p} f_{w}\left(\boldsymbol{r}, \boldsymbol{p},\left\{n_{q}\right\},\left\{n_{q}^{\prime}\right\}, t, \omega\right) \\
& \quad=\sum_{q^{\prime}} F\left(\boldsymbol{q}^{\prime}\right)\left\{e^{i\left(q^{\prime} \boldsymbol{r}-\omega_{q^{\prime}} t\right)} \sqrt{n_{q^{\prime}}+1}\right. \\
& f_{w}\left(\boldsymbol{r}, \boldsymbol{p}-\hbar \boldsymbol{q}^{\prime} / 2,\left\{n_{1}, \ldots, n_{q^{\prime}}+1, \ldots\right\},\left\{n_{q}^{\prime}\right\}, t, \omega-\omega_{q^{\prime}} / 2\right) \\
& -e^{-i\left(q^{\prime} \boldsymbol{r}-\omega_{q} t\right)} \sqrt{n_{q^{\prime}}} \\
& \quad f_{w}\left(\boldsymbol{r}, \boldsymbol{p}-\hbar \boldsymbol{q}^{\prime} / 2,\left\{n_{1}, \ldots, n_{q^{\prime}}-1, \ldots\right\},\left\{n_{q}^{\prime}\right\}, t, \omega+\omega_{q^{\prime}} / 2\right) \\
& +e^{-i\left(\boldsymbol{q}^{\prime} \boldsymbol{r}-\omega_{q^{\prime}} t\right)} \sqrt{n_{q^{\prime}}^{\prime}+1} \\
& \quad f_{w}\left(\boldsymbol{r}, \boldsymbol{p}-\hbar \boldsymbol{q}^{\prime} / 2,\left\{n_{q}\right\},\left\{n_{1}^{\prime}, \ldots, n_{q^{\prime}}^{\prime}+1, \ldots\right\}, t, \omega-\omega_{q^{\prime}} / 2\right) \\
& -e^{i\left(q^{\prime} \boldsymbol{r}-\omega_{q^{\prime}} t\right)} \sqrt{n_{q^{\prime}}^{\prime}} \\
& \left.\quad f_{w}\left(\boldsymbol{r}, \boldsymbol{p}+\hbar \boldsymbol{q}^{\prime} / 2,\left\{n_{q}\right\},\left\{n_{1}^{\prime}, \ldots, n_{q^{\prime}}^{\prime}-1, \ldots\right\}, t, \omega+\omega_{q^{\prime}} / 2\right)\right\}
\end{aligned}
$$

By collecting the above results we obtain the integro-differential equation for the WF of interest:

$\frac{\partial}{\partial t} f_{w}\left(\boldsymbol{r}, \boldsymbol{p},\left\{n_{q}\right\},\left\{n_{q}^{\prime}\right\}, t, \omega\right)+\frac{\boldsymbol{p}}{m} \frac{\partial}{\boldsymbol{r}} f_{w}+\boldsymbol{F} \frac{\partial}{\partial \boldsymbol{p}} f_{w}=\left.\frac{\partial f_{w}}{\partial t}\right|_{V}+\left.\frac{\partial f_{w}}{\partial t}\right|_{e p}$

where $\left.(\partial / \partial t)\right|_{V} f_{w}\left(\boldsymbol{r}, \boldsymbol{p},\left\{n_{q}\right\},\left\{n_{q}^{\prime}\right\}, t, \omega\right)$ is given by Eq. (3), and $\left.(\partial / \partial t)\right|_{e p} f_{w}\left(\boldsymbol{r}, \boldsymbol{p},\left\{n_{q}\right\},\left\{n_{q}^{\prime}\right\}, t, \omega\right)$ is given by Eq. (4).

The left-hand-side of the above equation is the standard Liouvillian operator of the Boltzman transport equation. This allows the standard use of path variables to transform the Wigner transport equation into its integral form. Considering for simplicity only a linear external potential besides the interaction with optical phonons we have:

$$
\begin{aligned}
& f_{w}\left(\boldsymbol{r}, \boldsymbol{p},\left\{n_{q}\right\},\left\{n_{q}^{\prime}\right\}, t, \omega\right)=f_{w}\left(\boldsymbol{r}_{o}, \boldsymbol{p}_{o},\left\{n_{q}\right\},\left\{n_{q}^{\prime}\right\}, t=0, \omega\right)+\int_{o}^{t} d t^{\prime} \sum_{q^{\prime}} F\left(\boldsymbol{q}^{\prime}\right) \\
& \left\{e^{i\left(\boldsymbol{q}^{\prime} \boldsymbol{r}\left(t^{\prime}\right)-\omega_{q^{\prime}} t^{\prime}\right)} \sqrt{n_{q^{\prime}}+1} f_{w}\left(\boldsymbol{r}\left(t^{\prime}\right), \boldsymbol{p}\left(t^{\prime}\right)-\hbar \boldsymbol{q}^{\prime} / 2,\left\{n_{1}, \ldots, n_{q^{\prime}}+1, \ldots\right\},\left\{n_{q}^{\prime}\right\}, t^{\prime}, \omega-\omega_{q^{\prime}} / 2\right)\right. \\
& -e^{-i\left(\boldsymbol{q}^{\prime} \boldsymbol{r}\left(t^{\prime}\right)-\omega_{q^{\prime}} t^{\prime}\right)} \sqrt{n_{q^{\prime}}} f_{w}\left(\boldsymbol{r}\left(t^{\prime}\right), \boldsymbol{p}\left(t^{\prime}\right)-\hbar \boldsymbol{q}^{\prime} / 2,\left\{n_{1}, \ldots, n_{q^{\prime}}-1, \ldots\right\},\left\{n_{q}^{\prime}\right\}, t^{\prime}, \omega+\omega_{q^{\prime}} / 2\right) \\
& +e^{-i\left(\boldsymbol{q}^{\prime} \boldsymbol{r}\left(t^{\prime}\right)-\omega_{q^{\prime}} t\right)} \sqrt{n_{q^{\prime}}^{\prime}+1} f_{w}\left(\boldsymbol{r}\left(t^{\prime}\right), \boldsymbol{p}\left(t^{\prime}\right)-\hbar \boldsymbol{q}^{\prime} / 2,\left\{n_{q}\right\},\left\{n_{1}^{\prime}, \ldots, n_{q^{\prime}}^{\prime}+1, \ldots\right\}, t^{\prime}, \omega-\omega_{q^{\prime}} / 2\right) \\
& \left.-e^{i\left(\boldsymbol{q}^{\prime} \boldsymbol{r}\left(t^{\prime}\right)-\omega_{q^{\prime}} t\right)} \sqrt{n_{q^{\prime}}^{\prime}} f_{w}\left(\boldsymbol{r}\left(t^{\prime}\right), \boldsymbol{p}\left(t^{\prime}\right)+\hbar \boldsymbol{q}^{\prime} / 2,\left\{n_{q}\right\},\left\{n_{1}^{\prime}, \ldots, n_{q^{\prime}}^{\prime}-1, \ldots\right\}, t^{\prime}, \omega+\omega_{q^{\prime}} / 2\right)\right\}
\end{aligned}
$$




\section{$E=0 t=50 \mathrm{fs}$}

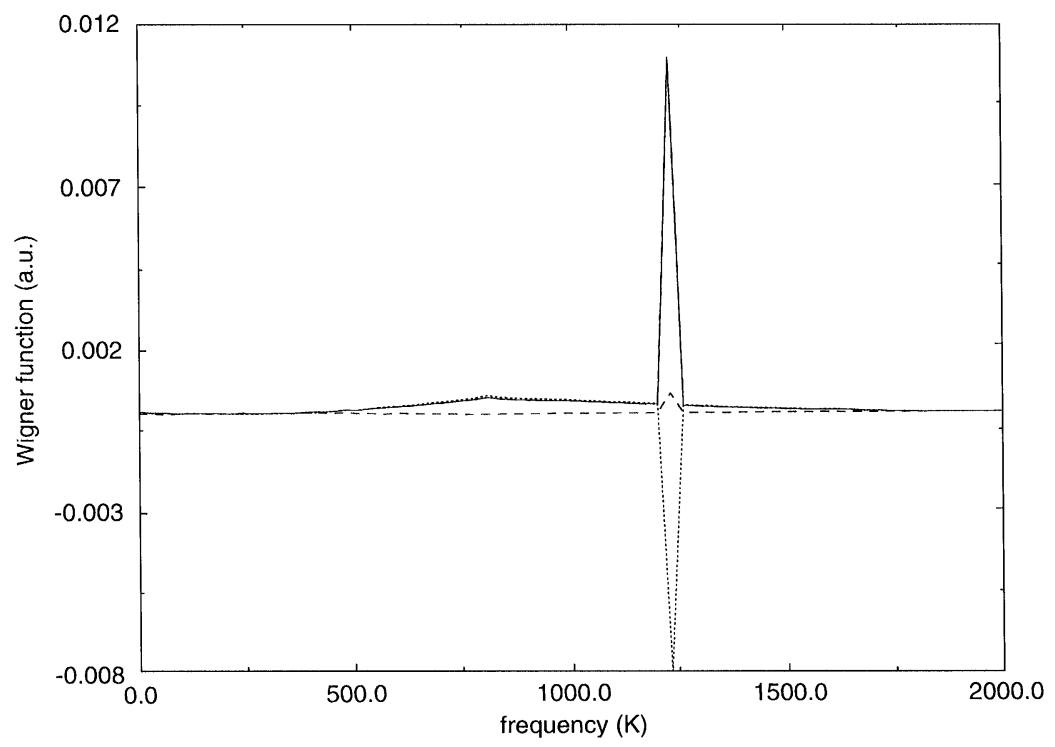

FIGURE 1 Wigner function as a function of frequency in absence of external fields at $T=300 \mathrm{~K}$ and $\mathrm{t}=50 \mathrm{fs}$ for a fixed value of $\mathbf{p}$. Continuous line represents the sum of the zero-order term of the Neumann expansion plus the corrections coming from paths with one (dotted line) and two (dashed line) optical-phonon scatterings.

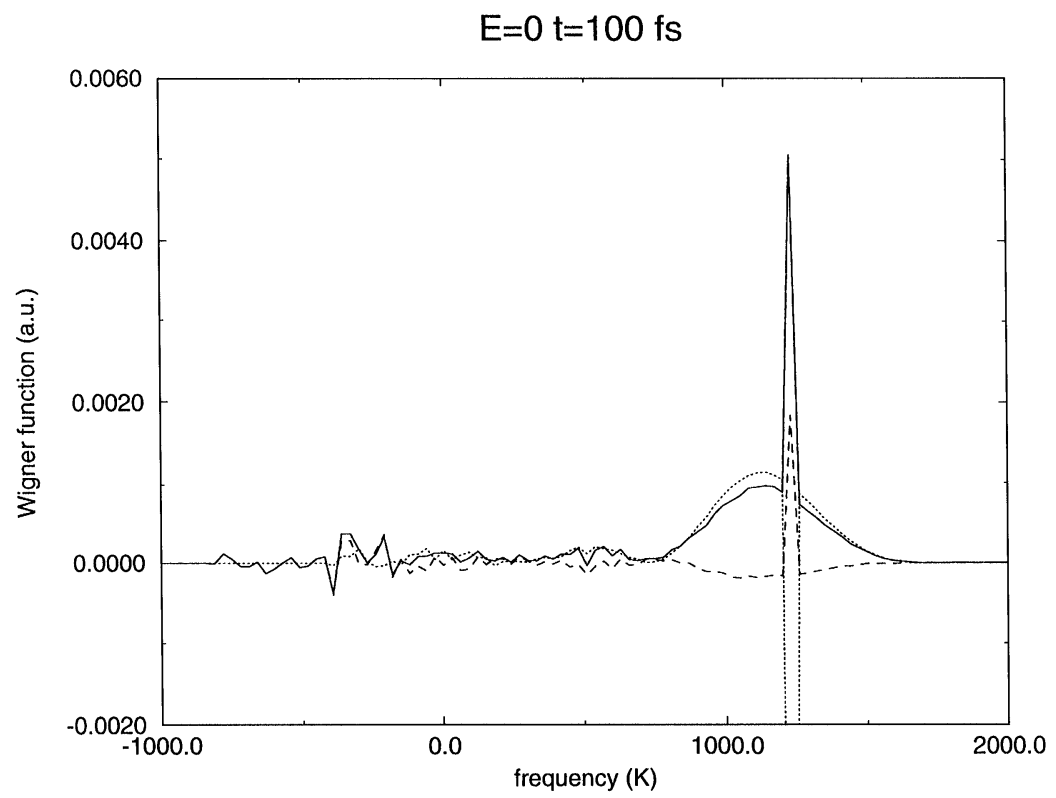

FIGURE 2 Wigner function as a function of frequency in absence of external fields at $T=300 \mathrm{~K}$ and $\mathrm{t}=100 \mathrm{fs}$ for the same value of p used in Figure 1. Continuous line represents the sum of the zero-order term of the Neumann expansion plus the corrections coming from paths with one (dotted line) and two (dashed line) optical-phonon scatterings. 


\section{SIMULATIVE APPROACH WITH WIGNER PATHS}

Following the approach already developed for the single-time WF, the above equation must be substituted into itself to obtain the perturbative Neumann series with terms at arbitrary perturbative orders.

The concept of Wigner path mentioned above is still applicable and allows to extend the Monte Carlo procedure based on the generation of WP already used to evaluate the single-time WF [4] for the calculation of $f_{w}\left(\boldsymbol{r}, \boldsymbol{p},\left\{n_{q}\right\},\left\{n_{q}^{\prime}\right\}, t, \omega\right)$. The only requirement to be added in the sequence of random choices and operations which define a particular path [4] is that at each electron - phonon interaction vertex half of the phonon frequency (besides half of the phonon momentum) is either added or substracted to the electron energy according to the selected integrand in the rhs of Eq. (5).

If the simulation is performed backward in time, the final $(r, p, \omega)$ is first chosen, where the WF will be evaluated, the path is evaluated backward in time and at the end of this evaluation the initial point of the WP, where the initial WF will be "read", is obtained.

As an example $f_{w}\left(\boldsymbol{r}, \boldsymbol{p},\left\{n_{q}\right\},\left\{n_{q}^{\prime}\right\}, t, \omega\right)$ has been calculated from a Monte Carlo calculation including paths with one and two phonon scattering in absence of external fields. Figures 1 and 2 show the results obtained at $t=50$ and $t=100 \mathrm{fs}$ as functions of $\omega$ assuming an equilibrium maxwellian initial condition for the WF and a fixed value for $\boldsymbol{p}$. It is shown that the WF is broadened in the energy domain and the effect is increasing at increasing times, while the zero-order peak is reduced by the negative contribution coming from the virtual (scattering-out) terms. For a classical particle the result would be a $\delta$ function centered at the frequency corresponding to $p^{2} / 2 m$.

\section{Acknowledgments}

This work has been partially supported by O.N.R. (Contract n. N00014-98-1-07777) and by the Italian Ministry for University and Scientific and Technological Research (Project MURST 40\% 1996).

\section{References}

[1] Bertoni, A., Bordone, P., Brunetti, R. and Jacoboni, C. (1999). "The Wigner function for electron transport in mesoscopic systems", J. Phys.: Condens. Matter, 11, $5999-6012$.

[2] Bertoni, A., Bordone, P., Brunetti, R., Jacoboni, C. and Sano, N. (1999). "Quantum versus classical scattering in semiconductor charge transport: a quantitative comparison", Physica B, 272, 299-301.

[3] Bordone, P., Pascoli, M., Brunetti, R., Bertoni, A. and Jacoboni, C. (1999). "Quantum transport of electrons in open nanostructures with the Wigner-function formalism", Phys. Rev. B, 59, 3060-3069.

[4] Pascoli, M., Bordone, P., Brunetti, R. and Jacoboni, C. (1998). "Wigner paths for electrons interaracting with phonons", Phys. Rev. B, 58, 3503-3506.

[5] Rossi, F., Jacoboni, C. and Nedjalkov, M. (1994). "A Monte Carlo solution of the Wigner transport equation", Semicond. Sci. Technol., 106, 121. 

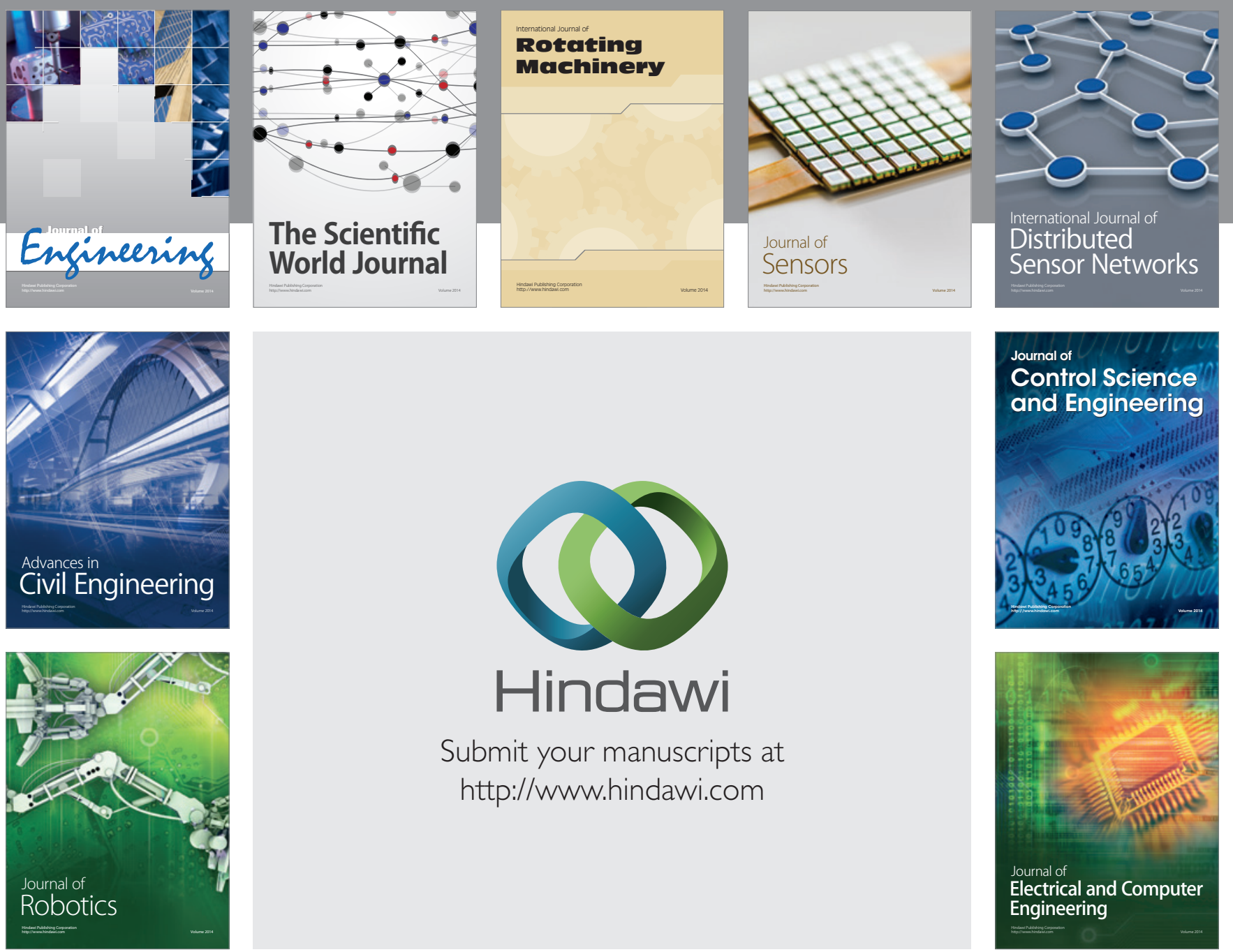

Submit your manuscripts at

http://www.hindawi.com
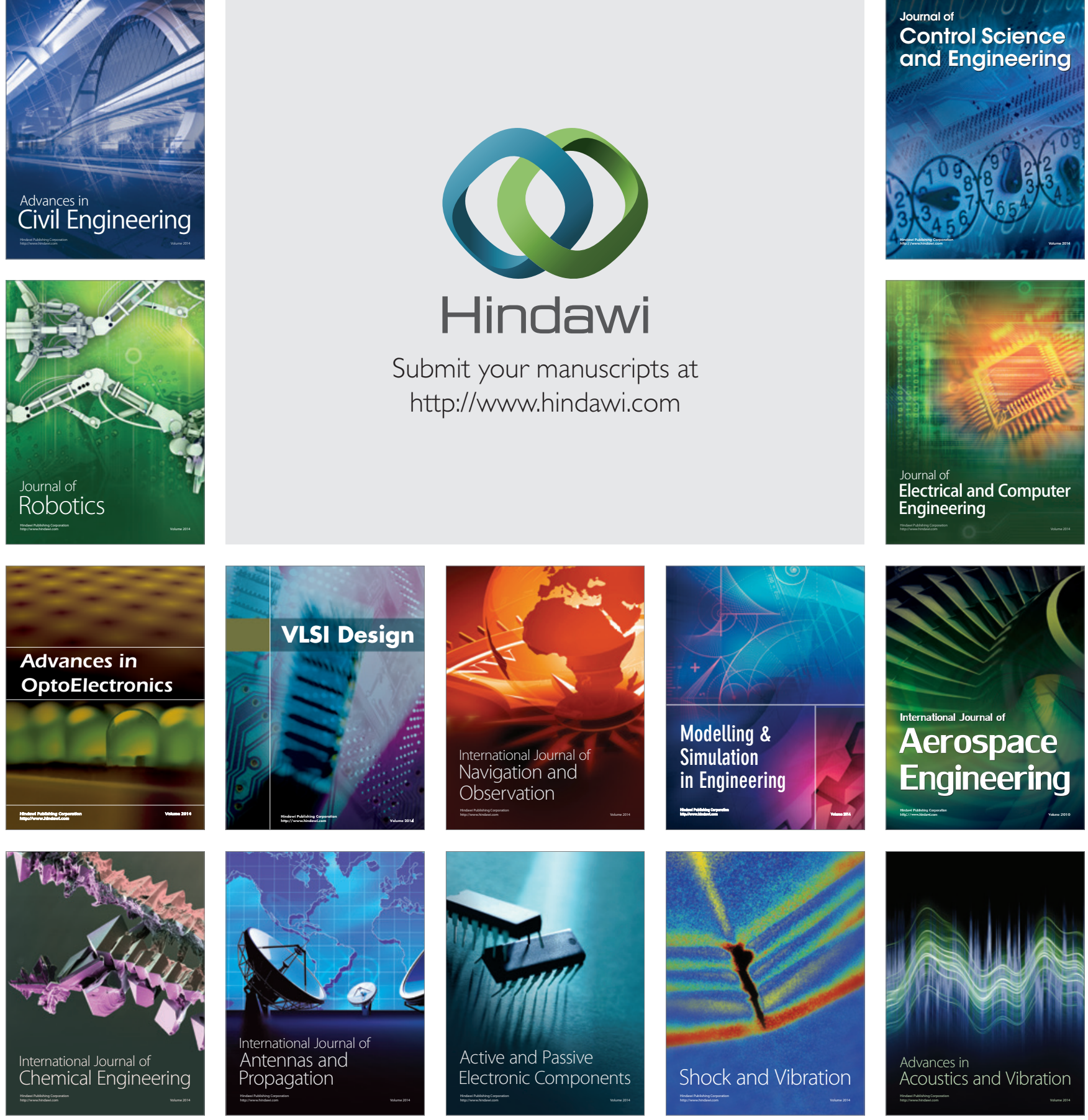